\title{
Hacia la formación de una conciencia feminista en Chile: antecedentes históricos, 1913-1952*
}

\author{
Claudia Rojas Mira
}

DEBATE FEMINISTA

Este trabajo se refiere al proceso de obtención de los derechos políticos plenos de las mujeres chilenas, proceso en el que ellas fueron activas protagonistas. Aborda, además, la conformación de una conciencia feminista en un periodo de casi medio siglo (1913-1952).

INTRODUCCIÓN

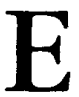
n los últimos 20 años, Chile ha visto surgir un movimiento de mujeres que en forma paulatina se ha incorporado a distintos ámbitos del acontecer nacional por medio de

* Una primera versión de este artículo fue presentada en el IV Encuentro de Historiadores Jóvenes, Santiago de Chile, Facultad Latinoamericana de Ciencias Sociales (FLACSO), 10 a 12 de abril de 1986. Dicha versión se tituló "La mujer chilena como fenómeno político de nuestro tiempo" y fue escrita en coautoría con mis amigas y colegas Edda Gaviola Artigas, Ximena Jiles Moreno y Lorella Lopresti Martínez. organizaciones, movilizaciones y cargos de representación popular.

Este protagonismo influye en el plano académico porque las mujeres comienzan a ser objeto de estudio en las distintas áreas del conocimiento. Algunas investigaciones, sobre todo las de carácter sociológico y psicológico, nos presentan a las mujeres desde ciertos rasgos específicos de sus vidas, aportando radiografias de su situación en el Chile de hoy, sin considerar sus antecedentes históricos y, por ende, los cambios que ha experimentado la participación de las mujeres a través del tiempo. Para la historiografia na- 
Secuencia (1996), 36, septiembre-diciembre, 245-248

ISSN: 0186-0348, ISSN electrónico: 2395-8464

DOI: http://dx.doi.org/10.18234/secuencia.v0i36.553

cional se trata de un terreno poco explorado, incluso en la actualidad. Las publicaciones que han enfocado la problemática de las mujeres desde una perspectiva histórica son escasas. ${ }^{1}$ Sin embargo, desde la década de los setenta, la historiografia internacional considera la participación de las mujeres ya sea en movimientos sociales o como actrices políticas en el devenir histórico. ${ }^{2}$

Este avance fue posible gracias a la renovación de la historia, al orientarse a superar la parcelación (historia económica, política o social) y al ocuparse de actores y espacios sociales antes considerados "sin historia". ${ }^{3}$ Dicha transformación se manifiesta, a partir de las últimas décadas, en el desarrollo de una historia social donde ha tenido ca. bida la historia de las mentalidades y la historia de las mujeres; en Estados Unidos y en Europa, ésta última es considerada un campo de investigación importante ${ }^{4}$ y sólo recientemente en América Latina se está abriendo un espacio para su desarrollo. Tardanza debida a la permanencia de una concepción androcéntrica de la historia en la que se privilegia la esfera pública y

1 "Hablamos de historia de las mujeres y no de historia de la mujer, pues la mujer en abstracto no tiene existencia histórica concreta en un tiempo y en un espacio". García, Problemas, 1994, p. 7. Véanse los pocos títulos: Labarca, Feminismo, 1947; Caffarena, Capítulo, 1952; Klimpel, La Mujer, 1962; Kirkwood, "La Política", 1984; Gaviola et al., "Queremos", 1986; Salinas, La Mujer, 1987; Hutchison, "El Feminismo", 1993.

2 Ramos, Género, 1992, p. 10.

3 Thompson, Miseria, 1981.

${ }^{4}$ Wallach Scott, Género, 1992, pp. 40-43, y Duby y Perrot, Historia, 1991, vol. 1, pp. 7-17. política asociada tradicionalmente con el mundo masculino, en donde es dificil hallar a las mujeres pues su espacio tradicional ha sido el hogar. Con anterioridad, sólo la experiencia masculina era valorada, lo que ha provocado la existencia de una visión fragmentada de la vida humana que ha dejado fuera la especificidad de la experiencia femenil. 5

${ }^{5}$ Para referirme a las mujeres como sujetos históricos usaré el término "femenil" en vez de "femenino", pues este último tiene connotaciones que refieren a débil, endeble, sumiso, pasivo, etcétera. Se conceptualiza lo femenino como minusvalía de las mujeres frente a los varones. Para el propósito de este trabajo utilicé las siguientes definiciones:

Femenil (del lat. femina, hembra, mujer): Adj. Propio de las mujeres.-Acad., Gran, 1980, vol. 2, p. 1216.

Femenino, (na) (del lat. femeninus): Adj. Propio y peculiar de las mujeres.// Aplícase al ser dotado de órganos para ser fecundado.// Perteneciente a este ser, o concerniente a él.// Fig. Débil, endeble.// Gram. Dícese del género gramatical, del nombre que significa mujer o animal hembra y del que se considera comprendido en este mismo género por su terminación, por su etimología o por el uso. U.t.c.s.// Gram. perteneciente o relativo a este género gramatical.-Acad., op. cit., 1980, vol. 2, p. 1216.

Feminismo: $[\ldots]$ no es una institución ni parece ser un asunto de conocimiento formal [...] De Lauretis, "Estudios", 1991, p. 168. [...] Existen tradiciones de pensamiento femenino, de cultura de las mujeres y de conciencia femenina que no son feministas...L fe femenino somos nosotras mismas, nuestros cuerpos y nuestra experiencia socialmente construida. Pero eso no es lo mismo que feminismo. El feminismo no es un producto 'natural' de aquella experiencia, sino una interpretación polémica y una lucha política que de ninguna manera se ha generalizado entre las mujeres. Linda Gordon, citado en De Lauretis; "Estudios", 1991, p. 171. [...] el feminismo se define a sí mismo como una instancia política; no se propone únicamente como una política sexual, sino como una política de la 
Cuando se estudia a las mujeres con un enfoque tradicional, ellas aparecen como caso insólito. Se trata de una historia de mujeres notables que deja en el anonimato a la gran mayoría. Otro tipo de estudios rescata a las mujeres desde su relación con hombres importantes, ya sea en una experiencia amorosa o en una empresa política. La debilidad de estos enfoques es cuantitativa y cualitativa, en el sentido de que omiten a la mitad de la población $y$ perciben a la otra como neutra. ${ }^{6}$

Las acciones y las experiencias históricas son concretas y muy diferentes de [las de] los seres humanos femeninos y masculinos, es necesario distinguir a la humanidad, entre otras categorías en hombres y mujeres, aquí la investigación histórica sobre las mujeres tiene su legitimación fundamental. ${ }^{7}$

Si bien su exclusión no ha sido única, ya que junto a ellas están los indígenas, los negros, los esclavos, los homosexuales, entre otros, las mujeres han formado parte de todas las socie-

experiencia, de la vida cotidiana, que posteriormente ingresa a la esfera pública de la expresión y de la práctica creativas, desplazando jerarquias estéticas y categorias genéricas y estableciendo así el fundamento semiótico de una producción diferente de referencias y significados. Ibid., 1991, p. 179.

Movimiento feminista: [...] es el que se convoca a partir de demandas de género, esto es, la denuncia y superación de la subordinación de las mujeres en la sociedad [...] De Barbieri, Movimientos, 1986, p. 13. Movimiento de mujeres: [...] son todos los que tienen una base de movilización mayoritariamente de mujeres, con independencia de sus demandas [...] Ibid., 1986, p. 13.

6 Radkau, Nueva, 1986 , p. 79.

${ }^{7}$ Ibid., 1986 , p. 80. dades y de todas las clases sociales a través de la historia de la humanidad. Han sido, tradicionalmente, las encargadas de la reproducción biológica, de resolver las múltiples necesidades de la vida cotidiana y de la socialización de niños y niñas. Por tanto, "escribir la historia de las mujeres supone tomarlas en serio, otorgar a las relaciones entre los sexos un peso en los acontecimientos o en la evolución de las sociedades". ${ }^{8}$

La renovación consiste en rescatar el papel de las mujeres en la historia, abordando los diversos aspectos de sus vidas: en la sexualidad, en el ámbito reproductivo, en el matrimonio y los nacimientos, en el cuidado de los niños y de la casa, en la alimentación, en el trabajo en el hogar y fuera de él, en los primitivos talleres artesanales, en la incipiente industria textil y del tabaco, en el ejercicio de la prostitución; en las cocinas y en las calles de las ciudades y los campos de América Latina. Estas temáticas podrían ser abordadas con un enfoque histórico que construya visiones complejas, donde lo público y lo privado aparezcan formando parte de lo social y lo político.

Cada uno de los periodos de la historia latinoamericana presenta sugerentes temas de investigación, fuentes y problemas diversos que podrían ser analizados desde esa mirada. En particular, el siglo Xx contiene rasgos de gran interés, ya que hay un incremento de la participación política, social y laboral de las mujeres. ${ }^{9}$

8 Duby y Perrot, Historia, 1991, p. 11.

${ }^{9}$ Es precisamente en el siglo $\mathrm{xx}$ cuando las mujeres del continente americano obtienen el derecho a sufragio pleno. Algunos ejemplos 
Importa abordar la realidad chilena como un caso de estudio notable, ya que en la mayor parte de los países las mujeres no obtuvieron el voto sino que lo recibieron "a modo de regalo" de los lideres políticos; en Chile, en cambio, hubo un movimiento organizado de mujeres que luchó por dicha conquista. De igual modo, la mayor participación de las mujeres chilenas en las elecciones, en lo que se refiere a la práctica del voto, proporciona otra motivación: "En Chile los índices de abstencionismo de las mujeres fueron constantemente más bajos que los de los hombres". ${ }^{10}$

De acuerdo con lo que plantea Chaney, dicho comportamiento se debió al marcado espíritu cívico de los chilenos, a que la política fue una actividad respetada y al vínculo del Estado con la Iglesia, la cual propició la participación y la ordenación ciudadana. Éstas últimas fueron canalizadas a través de los partidos políticos (que en Chile ejercen una fuerte influencia en los acontecimientos debido a su grado de organización). A su vez, la manera en que se estructuró el sistema político institucional permitió que los partidos integraran alianzas y coaliciones, y que entraran en juegos político-electorales competitivos, permitiendo con ello

son: Estados Unidos, 1920; Ecuador, 1929; Brasil, 1932; Uruguay, 1932; Cuba, 1933; Argentina, 1947; Venezuela, 1947; Costa Rica, 1949; Chile, 1949; México, 1953; Nicaragua, 1955; Perú, 1955; Colombia, 1957; Paraguay, 1961. Esto implicó un incremento de su participación, puesto que la legalidad amplió las posibilidades de las mujeres para participar en un ámbito, con anterioridad, de exclusiva participación masculina, OEA, Comision, 1965, p. 17.

${ }^{10}$ Chaney, Supermadre, 1983, p. 144. la expresión ciudadana institucionalizada.

Chile proporciona un laboratorio electoral único para cualquiera que se interese en estudiar el comportamiento electoral de hombres y mujeres. Cuando éstas votaron por primera vez en las elecciones municipales, se estableció un registro electoral separado (junto al de los extranjeros) ${ }^{11}$ que se convertiría en una práctica, por así decirlo, consuetudinaria. Además de los registros electorales separados, hubo locales y recuentos de votación por sexo. Por tanto, Chile es uno de los pocos países que posee estadísticas electorales por sexo.

Antes de entrar en materia quisiera plantear algunas reflexiones acerca de la participación política de las mujeres y la democracia. El acceso de las mujeres a la esfera pública significa la obtención de un espacio en el ámbito educativo, laboral y político, contribuyendo en cierto sentido a su liberación. ${ }^{12}$ Sin embargo, me pregunto si efectivamente estos progresos permiten su emancipación y acceso al poder, como para originar una transformación de la sociedad en la que hombres y mujeres puedan desarrollarse integralmente. En respuesta a lo anterior, está el reducido número de mujeres que ingresa a las esferas del poder público (ejecutivo, legislativo,

${ }^{11}$ El registro municipal incluyó a mujeres y extranjeros hasta 1947; con anterioridad a esa fecha no se tienen datos desagregados de participación femenil en las elecciones municipales.

${ }^{12}$ La liberación de las mujeres es el proceso a través del cual éstas se constituyen en personas autónomas y con igualdad de oportunidades en la sociedad. 


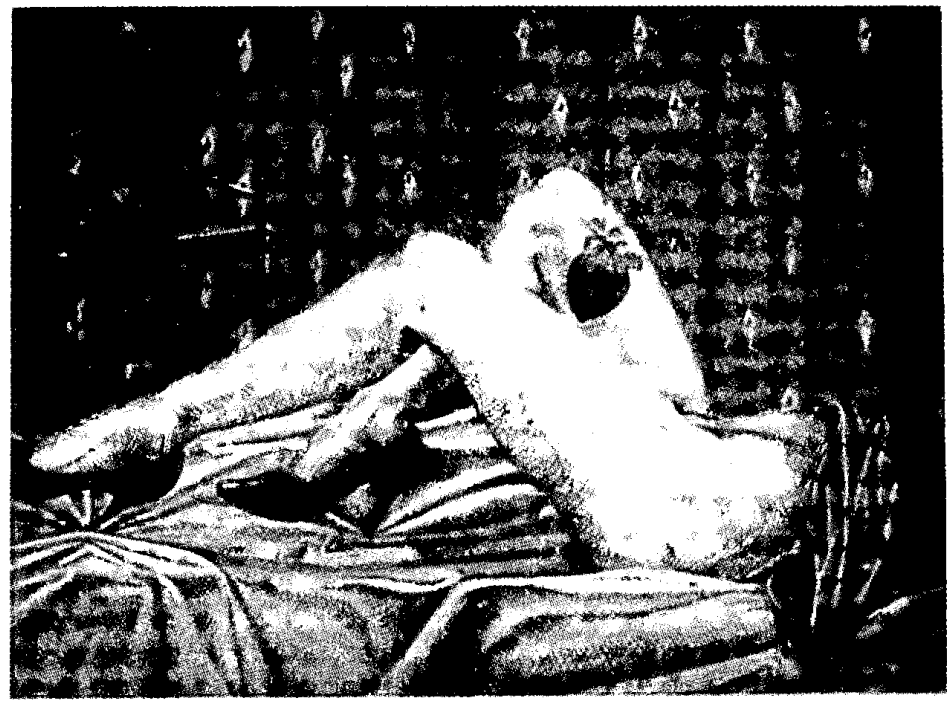

judicial, partidos políticos, entre otras) tradicionalmente masculinas. Este escaso número se conforma, salvo excepciones, por mujeres privilegiadas en cuanto a educación, dinero, posición social o tipo de trabajo que les toca desempeñar. Dicha diferencia cuantitativa tiene su paralelo en lo cualitativo, pues existe un "modelo masculino" y un "modelo femenino" de ejercicio del poder que se relaciona con la socialización política que experimentan hombres y mujeres, y el sexismo que la sustenta. En-tenderemos por socialización política:

todo tipo de aprendizaje político, formal e informal, deliberado o no, en todos los estadios del ciclo vital, incluyendo no sólo el aprendizaje político explícito, sino también el nominalmente político, que afecta sin embargo el comportamiento político. ${ }^{13}$

Por su parte, el sexismo consiste en la discriminación que se hace en función del sexo de una persona, como clasismo en la discriminación por clase y racismo en la discriminación por raza. Históricamente se ha discriminado a las mujeres por razón de su sexo. El sistema social en el cual estamos insertos (capitalismo) es clasista, racista y sexista. El "machismo" es una vertiente del sexismo y es una expresión agresiva y burda que coloca a los hombres como mejores y destinados a ciertos papeles dentro de la sociedad y a las mujeres como subordinadas.

13 Gallego, Nuevas, s/f., p. 43. 
El "modelo masculino" está asociado a lo exclusivo, excluyente, autoritario, jerárquico, heroico, temerario, competitivo, destructivo, por lo cual "nuestra participación en lo público no cuestiona por sí sola este modelo de poder". ${ }^{14}$ Respondiendo a esta socialización diferenciada, se espera que las mujeres seamos, en la función pública, laboriosas, oscuras, no ambiciosas, serviciales, apoyadoras, diligentes, sensibles socialmente y que aportemos a la política el tono moral. Es cierto que en este ámbito ejercemos un protagonismo, pero ¿de cuál protagonismo se trata? Aquél en el que nos hacemos visibles porque sobre nuestros hombros cargamos los papeles y la ideología que nos impuso la familia, es decir, como madres, esposas y amas de casa (movilizándonos, por ejemplo, contra la carestía de la vida) o, por el contrario, porque nos acercamos al modelo masculino mediante un proceso de "masculinización" (el caso de Margaret Thatcher), asumiendo rasgos y características específicas para alcanzar puestos de elección. No obstante, entre estas dos formas polares de participación de las mujeres surge otra, especialmente en América Latina, que desdibuja los patrones tradicionales practicando la política en "condiciones y con particularidades específicas que no coinciden con la concepción y la práctica de la política basada en el modelo masculino, - porque- pone en cuestión los límites entre lo político y lo no político", 15 es

14 Vargas, Revista, 1986, p. 11.

15 Bonder, Revista, 1983 , p. 616 . decir entre la esfera de lo público y la de lo privado.

De tal manera que las relaciones de pareja, la sexualidad, el papel materno, la vida familiar, la reproducción, la práctica del aborto, el uso de métodos anticonceptivos y el divorcio, antes incuestionables o reducidos al ámbito privado, aparecen hoy como relaciones de poder y se amplía el significado de la acción política. El poder aparece atravesando toda la trama social y las relaciones interpersonales, es decir, la vida cotidiana. ${ }^{16}$

El accionar político de las mujeres en la historia de América Latina se ha legitimado por medio de su papel materno, independientemente del tipo de opción político-partidaria. ${ }^{17}$ Basta recordar las movilizaciones de las mujeres en Brasil y Chile ("marcha de las cacerolas vacías"), contra gobiernos progresistas, en nombre de la familia y los hijos, o las movilizaciones de las Madres de la Plaza de Mayo en Argentina, o las reivindicaciones económicas, políticas y sociales -que adquieren significación a partir de la relación madre-hija(o). Estas prácticas han transformado la manera tradicional de hacer política, incluyendo elementos y contenidos nuevos antes considerados no políticos o pertenecientes al ámbito privado y cuya resolución se daba en el interior del mismo. ${ }^{18}$

La mujeres han participado en politica antes y después de la obtención de su derecho al sufragio pleno (dentro y fuera de la política formal). A raíz de

\footnotetext{
16 Foucault, Microfísica, 1979.

17 Chaney, Supermadre, 1983.

${ }^{18}$ Kirkwood, Revista, 1983.
} 
este hecho, su participación se ha legitimado a nivel institucional y, probablemente, su presencia ha implicado cambios en el ejercicio y en el significado de esta actividad, incidiendo en las decisiones importantes y cambiando con ello las relaciones de poder, por lo que se hace necesario estudiar dicho proceso.

En los debates académicos sobre democracia no se han tomado en cuenta las relaciones de género ni la desigualdad social que éstas conllevan. Tampoco se ha considerado que las desigualdades sociales desvirtúan las posibles igualdades en el ámbito político. ${ }^{19}$ No es posible considerar democrático a un sistema en el que hay una división expresa entre la vida pública y la privada, entre las actividades que desempeñan hombres y mujeres. Los debates académicos han redundado en la estructura clasista de la sociedad y la forma en que el clasismo impide una real igualdad política, y prácticamente no han analizado las desigualdades genéricas.

el fracaso del marxismo clásico en cuanto a la plena expresión o conceptualización de la opresión sexual [...], el marxismo como teoría de la vida social, prácticamente no está interesado en el sexo. En el mapa del mundo social de Marx, los seres humanos son trabajadores, campesinos o capitalistas; el hecho de que también son hombres o mujeres no es visto como muy significativo. En contraste, en los mapas de la realidad social trazados por Freud y Lévi-Strauss hay un profundo reconocimiento del lugar de la sexualidad en la sociedad, y

${ }^{19}$ Pateman, Debate, 1990. de las profundas diferencias entre la experiencia social de los hombres y la de las mujeres. ${ }^{20}$

Si bien es cierto que las mujeres han ganado el derecho al voto y otras garantías civiles, no es menos cierto que ello se da en el contexto de la subordinación social de éstas, con lo que se manifiesta una contradicción profunda entre la igualdad política formal que se verifica en el ámbito público y la discriminación de las mujeres en la esfera privada como esposas, madres y amas de casa. Como lo señala Pateman,

¿cómo pueden las mujeres y las esposas que han "elegido" la vida doméstica tener la oportunidad de desarrollar sus capacidades o de aprender lo que significa ser un ciudadano democrático? ${ }^{21}$

Las acciones y reflexiones del movimiento feminista han permitido incorporar a las teorías sobre la democracia dimensiones hasta hace un tiempo no consideradas. La afirmación de "lo personal es político" ha enriquecido el debate teórico sobre la democracia y ha abierto nuevas perspectivas sobre las relaciones humanas que implican relaciones de poder.

A continuación, daré una mirada general de lo que fueron las principales etapas e hitos históricos del movimiento feminista chileno hasta la consecución del voto y la primera elección presidencial con participación femenil (1913-1952).

${ }^{20}$ Rubin, Nueva, 1986, pp. 97-98.

${ }^{21}$ Pateman, Debate, 1990, p. 17. 
DESPERTAR DEL MOVIMIENTO FEMINISTA (1913-1925)

Si pudiera resumirse en una sola idea el sello característico de este periodo habría que mencionar, sin duda, el despertar de la conciencia feminista en Chile, expresado en la demanda que hicieran las maestras normalistas Antonia Tarragó e Isabel Le Brun, por el libre acceso de las mujeres a todos los niveles educacionales. Su petición no tardó en concretarse ya que en 1877 , gracias a la dictación del Decreto Amunátegui, se abrieron las puertas de la universidad para las mujeres chilenas. ${ }^{22}$ Este hecho fue decisivo en el despertar de la conciencia feminista $y$ en el nacimiento de una inquietud colectiva respecto del cuestionamiento del papel que las mujeres ocupaban dentro de la sociedad.

Otro factor vital de este fenómeno lo constituyeron las visitas, a nuestro país, que a partir de 1910 realizaron conferencistas extranjeros para referirse al tema del feminismo, entre quienes cabría mencionar a Adolfo Posada, ConcepciónJimeno de Flaquer y Belén de Sárraga. ${ }^{23}$ De todas ellas, fue sin duda la visita de esta última la que causó hondas repercusiones en la vida nacional. Belén de Sárraga recorrió Chile dictando charlas y enarbolando las banderas del libre pensamiento y del anticlericalismo; fue una de las primeras en hacer un llamado en favor de la emancipación de las muje-

${ }^{22}$ Maino et al., Tres, 1978, p. 212.

${ }^{23}$ El Mercurio de Santiago, El Despertar de los Trabajadores (Iquique) y El Mercurio de Valparaíso, 1913. res. Ilustraba en sus charlas lo que para ella era el origen de la postración de las mujeres, en palabras como éstas:

En un concilio del siglo vi de la era cristiana se sometió a discusión si la mujer tenía alma y sólo por dos votos a favor quedó resuelta afirmativamente esta duda. Calculad cuál habría sido la situación si aquellos dos santos varones por cualquier circunstancia llegan tarde a la votación. ${ }^{24}$

Se difundieron, también, a través de la prensa el desarrollo del feminismo a nivel mundial y las ideas de igualdad que postularon Stuart Mill, Federico Engels y Carlos Marx.

Estos antecedentes, sumados al impacto que provocó la incorporación de las mujeres a las diversas áreas de la producción durante la primera guerra mundial(1914-1918), dieron un impulso extraordinario al despertar femenil. Junto a esto, habían madurado en las propias mujeres y en su forma de vida las principales motivaciones que las llevaron a organizarse. Es así como el primer brote de organizaciones feministas fueron los Centros Femeninos Anticlericales "Belén de Sárraga" fundados en Iquique y en la zona salitrera a partir de 1913, bajo la inspiración de la propia Belén de Sárraga. Posteriormente el trabajo constante y tesonero de Luis Emilio Recabarren, ${ }^{25}$ Teresa Flores, Adela de Lafferte, Rosario de Barnés, entre otras, 1913.

24 El Mercurio de Valparaíso, 14 febrero

${ }^{25}$ Líder del movimiento obrero chileno y fundador, en 1912, del Partido Obrero Socialista, que posteriormente se llamó Partido Comunista. 
hicieron del norte la cuna del feminismo en Chile. ${ }^{26}$

Varios factores interrelacionados facilitaron dicho acontecimiento: en primer término, el desarrollo económico que alcanzó esta zona gracias a la explotación del salitre y a una gran concentración de población que dejó aflorar los problemas compartidos, contribuyendo así a la organización de las mujeres. Aquello provocó una mayor permeabilidad ante las influencias externas, acentuando la debilidad de los lazos tradicionales (comolos religiosos) en la sociedad que se desarrollaba en la Pampa.

Hay que contar, además, con el surgimiento del Partido Obrero Socialista (1912) -fundado por Luis Emilio Recabarren-y su constante labor periodística y agitativa a través de El Despertar de los Trabajadores, para dar a las mujeres un lugar preferente como lo demuestra la siguiente cita:

Si se quiere que la mujer coopere de un modo eficiente a la realización de las ideas de progreso y libertad que hoy por todas partes se propalan, debe educársela con esmero y redimírsela-como aspira el feminismo- de ese estado de postracióny servidumbre en que yace. ${ }^{27}$

La movilización de las mujeres en Iquique y la pampa fue de carácter reivindicativo: contra la carestía de la vida, por el derecho al descanso dominical de los obreros y contra el alcoholismo; pero también divulgaron las ideas de emancipación de las mujeres

${ }^{26}$ Poco antes se fundó un centro en Valparaíso con las mismas características, sin embargo, éste no prosperó. ElDespertar de los Trabajadores, Iquique, 1913.

27 Ibid., 30 abril 1912. a través de charlas y veladas-conferencias. De tal forma, estos centros feministas presentaron una clara ligazón con las corrientes políticas socialistas y anarquistas de la región, como también con el movimiento obrero que se desarrolló en la zona. Esto provocó, con el correr del tiempo, una migración de mujeres hacia el Partido Obrero Socialista y a los sindicatos, con la consiguiente pérdida de vigencia del movimiento feminista en cuanto tal. En lo sucesivo, fue la ciudad de Santiago desde donde partieron las más importantes iniciativas para mejorar la situación de las mujeres.

Mientras el movimiento feminista se encontraba más avanzado en Iquique, en Santiago y Valparaíso, en los mismos años (1913-1925) las mujeres de elite centraron su atención en la beneficencia pública: la Cruz Roja y la Fundación Gotas de Leche son muestra clara de ésto. No obstante, a partir de 1915 el Círculo de Lectura y el Club Social de Señoras fundados en Santiago, rompieron en alguna medida dicho esquema. Orientaron su labor a elevar el nivel cultural de sus asociadas.Jugóallí un papel importante quien más tarde se convertiría en una connotada dirigente feminista: Amanda Labarca (1886-1975). Sin embargo, no fue sino hasta principios de la década de 1920 cuando surgieron en Santiago organizaciones cuyo motor fue la lucha por la obtención de los derechos políticos; en este sentido cabe mencionar al Consejo Nacional de Mujeres (heredero del Círculo de Lectura), que en 1922 presentó un proyecto de ley sobre derechos civiles y políticos, que no fructificó. Ese mismo año se creó el 
Partido Cívico Femenino, en el cual destacaron figuras como Graciela Mandujano y Ester La Rivera de Sanhueza. En 1924 surgió el Partido Demócrata Femenino que se abocó a luchar por tales derechos. En éste, destacó Celinda Arregui, quien tuvo una actuación sobresaliente en la realización, en 1922, del Congreso Panamericano de Mujeres, que solicitó formalmente la concesión de los derechos políticos para las mujeres chilenas.

Entre 1915 y 1922 numerosos proyectos sobre derechos civiles y políticos fueron a dormir en los archivos del Congreso Nacional, esperando mejoresoportunidades para prosperar. Sólo en 1925 se concretaron dichas peticiones con la concesión de derechos civiles a las mujeres: la madre obtuvo la patria potestad al morir el padre, se permitió a las mujeres ser testigos en juicios y tutoras de menores, pero con autorización del marido si eran casadas, entre otros.

Así fue como, a través de estas iniciativas, la conciencia feminista y el espíritu asociativo despertó en las mujeres chilenas. Y junto a ellos, su deseo de obtener un lugar en la vida nacional. Fue el inicio de una larga lucha, en la cual las mujeres fueron madurando en su organización y movilizaciones, hasta llegar a exigir sus derechos ciudadanos.

ORGANIZACIÓN Y ACCIONES DEL MOVIMIENTO FEMINISTA (1926-1946)

Si el periodo anterior se caracterizó por el surgimiento de las primeras or- ganizaciones de mujeres en la historia de Chile, el desarrollo y la continuidad de algunas, como el Partido Cívico Femenino y el Consejo Nacional de Mujeres, y por el logro de una de las más grandes aspiraciones del movimiento feminista: la concesión de importantes derechos civiles a las mujeres en 1925, esta nueva etapa estuvo marcada por la creación y la lucha de las más importantes organizaciones que conoció la historia femenil hasta nuestros días: el Movimiento Pro Emancipación de la Mujer Chilena (MEMCH, 1935) y la Federación Chilena de Instituciones Femeninas (FECHIF, 1944); éstas incluyeron en sus programas, como aspecto fundamental, la lucha por la consecución de los derechos políticos de las mujeres.

Paralelamente a la fundación de la Asociación de Mujeres Universitarias en 1931 (la que además de su quehacer cultural planteó públicamente una lucha por los derechos políticos femeniles), ${ }^{28}$ la Liga de Damas Chilenas realizaba, en medio de la crisis económica de 1930, un llamado a combatì la desocupación y a proteger la industria nacional, mientras mujeres emparentadas con las víctimas políticas del gobierno del general Carlos Ibáñez del Campo realizaban un desfile de protesta en julio del mismo año. ${ }^{29}$

De carácter más enérgico y prolongado fue la acción que las mujeres de Chile emprendieron con motivo de la campaña presidencial de Juan Esteban Montero, candidato representante de la civilidad, en contraposición a las ar-

28 El Mercurio, 3 agosto 1931.

29 Ibid., 18 julio 1931. 
bitrariedades del gobierno de Carlos Ibáñez, constituyéndose esta lucha en un hito que marcó el inicio de la participación decidida de las mujeres en el plano político. Tal fue la energía que las mujeres evidenciaron en el Teatro Libertad, en septiembre de 1931, con motivo de la campaña de Montero, que el afamado escritor Rafael Maluenda escribió lo siguiente:

ha llegado una fuerza nueva. La asamblea de ayer marca una fecha en nuestros progresos democráticos. La mujer se adelanta y hace sentir su presencia en la vida cívica. Cierta de que nadie puede ahorrarle su parte de responsabilidad en los resultados, se alza para representar su opinión, que no es ya la opinión de una simple y cordial simpatía, sino la expresión de una convicción asumida a conciencia. ${ }^{30}$

El espíritu asociativo femenil dio origen en 1933 al Comité Nacional Pro-Derechos de la Mujer, creado a iniciativa de Felisa Vergara, con objeto de exigir del Parlamento la promulgación de una ley de voto municipal que, como retribución a las mujeres por el apoyo prestado, el propio Juan Esteban Montero había propuesto. La misma institución resurgió en 1941, una vez cumplido su objetivo, para exigir esta vez la posibilidad de votar en elecciones parlamentarias y presidenciales, conforme lo había sugerido el presidente Pedro Aguirre Cerda, mediante un proyecto de ley redactado por el MEMCH en enero del mismo año. ${ }^{31}$

${ }^{30} \mathrm{Ibid}$, 22 septiembre 1931.

31 Ibid., 20 agosto 1941.
Entre tanto, en 1935, durante el segundo gobierno de Arturo Alessandri Palma, en medio de una crisis económica de proporciones y tras el resultado de la primera elección municipal con participación femenil en que venció la derecha por abrumadora mayoría, surgió el Movimiento Pro-Emancipación de la Mujer Chilena (MEMCH) con el objeto de luchar por la emancipación económica, social, jurídica y política de las mujeres. Más aun, cuando ninguna institución se ocupaba de la sexualidad de las mujeres, el MEMCH pidió a las autoridades gubernamentales y científicas que se preocuparan por los repetidos embarazos que desembocaban en abortos clandestinos, enfermedades, miseria o muerte de los niños. ${ }^{32}$

El mérito del MEMCH, articulado en más de 60 filiales a lo largo del país, residió en su masividad y continuidad temporal, en las movilizaciones que sus militantes realizaron por el voto político, contra la carestía de las subsistencias, por el Día Internacional de la Mujer ( 8 de marzo), la paz, contra el fascismo y las relegaciones. ${ }^{33}$ Aunque se declaró como un movimiento am. plio desde el punto de vista social, político y religioso, representó en los hechos el brazo femenil del Frente Popular-alianza de centro-izquierda fundada en 1936, que congregó a los partidos Radical, Comunista y Socialista, entre otros, para llevar a la presidencia

${ }^{32}$ La Opinión, Santiago, 27 mayo 1935.

${ }^{33}$ La relegación es una acción represiva em. pleada por el gobierno, que consiste en expulsar a una persona de su comunidad, por razones políticas, y llevarla a un sitio apartado dentro del país durante un lapso determinado. 
de la república al militante radical Pedro Aguirre Cerda (1939-1941) sin por ello renunciar a su autonomía.

Así, otro de sus importantes logros fue la labor realizada en el Frente Popular con el propósito de que la izquierda cambiara su actitud indiferente $y$ temerosa ante el "problema femenino" y se mostrara más receptiva. En ese momentáneo cambio tuvo gran importancia un hombre que siempre estuvo del lado de las feministas: el militante del partido Radical Pedro Aguirre Cerda, tras cuya inesperada muerte, la indiferencia y el menosprecio hacia el sector femenil se ahondó hasta el punto de que su resolución constituye una de las necesidades más urgentes del momento actual: la necesidad de estrechar lazos entre los partidos de izquierda y las organizaciones feministas para no perder el voto del sector femenil, como ocurrió en las primeras elecciones en que participó. En relación con este punto, ya en 1940 el MEMCH sostuvo que el partido que mejor interpretara las aspiraciones de las mujeres se llevaría la mayor parte de los sufragios. ${ }^{34}$

Hasta la realización del Primer Congreso Nacional de Mujeres (Santiago, 1944), el movimiento femenil había conseguido muchos progresos, pero hacía falta una institución que aglutinara a las mujeres, específicamente en torno a la obtención del sufragio. La respuesta a esa necesidad fue la creación de la Federación Chilena de Instituciones Femeninas (FECHIF), hija del ya citado congreso y dirigida por Aman-

${ }^{34}$ El Siglo, 26 octubre 1940.

${ }^{35}$ El Mercurio, 8 noviembre 1944.

${ }^{36}$ El Stglo, 26 octubre 1946. da Labarca. Llevó a cabo una campaña intransigente por la obtención del voto, en la que estuvieron presentes los foros, los mensajes radiales y las manifestaciones multitudinarias en teatros y calles. ${ }^{35}$ Sus militantes apoyaron la candidatura presidencial de Gabriel González Videla pero sin perder por ello de vista sus propias reivindicaciones. ${ }^{36} \mathrm{El}$ popular eslogan Las mujeres no votamos pero el triunfo asegura. mos, de uso frecuente durante la campaña, demuestra el interés de estas mujeres por ligar la lucha electoral de su candidato a la lucha por sus derechos políticos plenos.

Además de la labor realizada por el MEMCH y la FECHIF, fue determinante en la obtención de los derechos políticos femeniles la incorporación de muchas mujeres a los partidos políticos. En ellos aprendieron a socializarse políticamente; sin embargo no alcanzaron, salvo excepciones (como las del Partido Conservador y el Partido Comunista) cargos dirigentes, dada la persistencia de una fuerte carga "machista" en estas organizaciones.

Coincidiendo con la creación de las organizaciones más importantes en la ciudad de Santiago, se dio en el puerto de Valparaíso la fundación de instituciones militantes como La Unión Femenina de Chile, en 1928. Ésta se creó básicamente para obtener los derechos políticos como único camino que lograría un mejoramiento integral de la situación de las mujeres. ${ }^{37}$ Esta organización, si bien no fue masiva, tuvo una importante influencia en la

${ }^{37}$ Dirección General de Estadísticas, "Estadística Chilena", agosto de 1935, p. 315. 
opinión pública de Valparaíso, sobre todo a través de dirigentes como Graciela Lacoste y de un órgano de prensa propio, el periódico La Unión Femenina de Chile, que se publicó entre 1934 y 1950.

Por su parte, el puerto de Iquique, un centro importante del feminismo chileno del periodo anterior, creó en estos años organizaciones como el MEMCH local, que se abocó a luchar por el mejoramiento de las condiciones de vida de las mujeres y sus familias, debido a la situación de miseria que se vivía en la pampa salitrera en esos años, y debiendo relegar a un segundo plano las reivindicaciones especificas del género.

Otro elemento vital en la obtención de la plenitud de los derechos políticos fue la conquista del voto municipal y su ejercicio en las elecciones de $1935,1938,1941$ y 1944 . Pero si las mujeres habían demostrado de tantas maneras su madurez e interés político, ¿por qué entonces no se les concedía derecho a sufragar en elecciones parlamentarias y presidenciales? Probablemente debido a un problema táctico: el comportamiento electoral femenil, tan temido por los partidos de la época, debía ser sometido a prueba con un bajo costo político porque no era conveniente arriesgar las elecciones más importantes como las de diputados, senadores o presidente de la república. La primera elección municipal con participación femenil arrojó un resultado favorable para la derecha. ${ }^{38}$ Se creó, entonces, el mito del

${ }^{38}$ Dirección General de Estadísticas, "Estadisticas Chilenas", 1944. conservadurismo de las mujeres, incorporado como un rasgo propio de nuestra "naturaleza". La tendencia marcadamente conservadora mostrada por el elemento femenil antes de 1941 tiene causas bien concretas: la influencia de la Iglesia católica y la familia tradicional, la eficiente labor de los medios de comunicación derechistas para atraerse a esta nueva fuerza política, el uso de sus medios económicos para financiar sus campañas electorales y el cohecho (compra y venta de sufragios), lo engorroso y caro del sistema electoral, que no permitía a las clases populares disponer ni del tiem. po ni del dinero necesarios para inscribirse en los registros electorales y materializar su voto. El ulterior cambio en los resultados ${ }^{39}$ se explicaría, por su parte, gracias a los lazos existentes entre el MEMCH y el Frente Popular, la labor desplegada por éste en favor de las mujeres, la fuerza que adquirió el Frente después del triunfo de su candidato y el desprestigio de la derecha tras el gobierno de Arturo Alessandri Palma.

Las elecciones municipales de 1935 , 1938,1941 y 1944 demostraron que el comportamiento electoral de las mujeres no es más de izquierda o más de derecha, ya que su comportamiento político se manifiesta de acuerdo con las circunstancias históricas que les haya tocado vivir.

Simultáneo al surgimiento de la conciencia feminista y la acción de las más

39 Entre 1935 y 1944 , el apoyo a la izquierda en las elecciones municipales creciô de $27.3 \%$ a $43.5 \%$, respectivamente. Dirección General de Estadísticas, "Estadisticas Chilenas", 1944. 
Secuencia (1996), 36, septiembre-diciembre, 245-248

ISSN: 0186-0348, ISSN electrónico: 2395-8464

DOI: http://dx.doi.org/10.18234/secuencia.v0i36.553

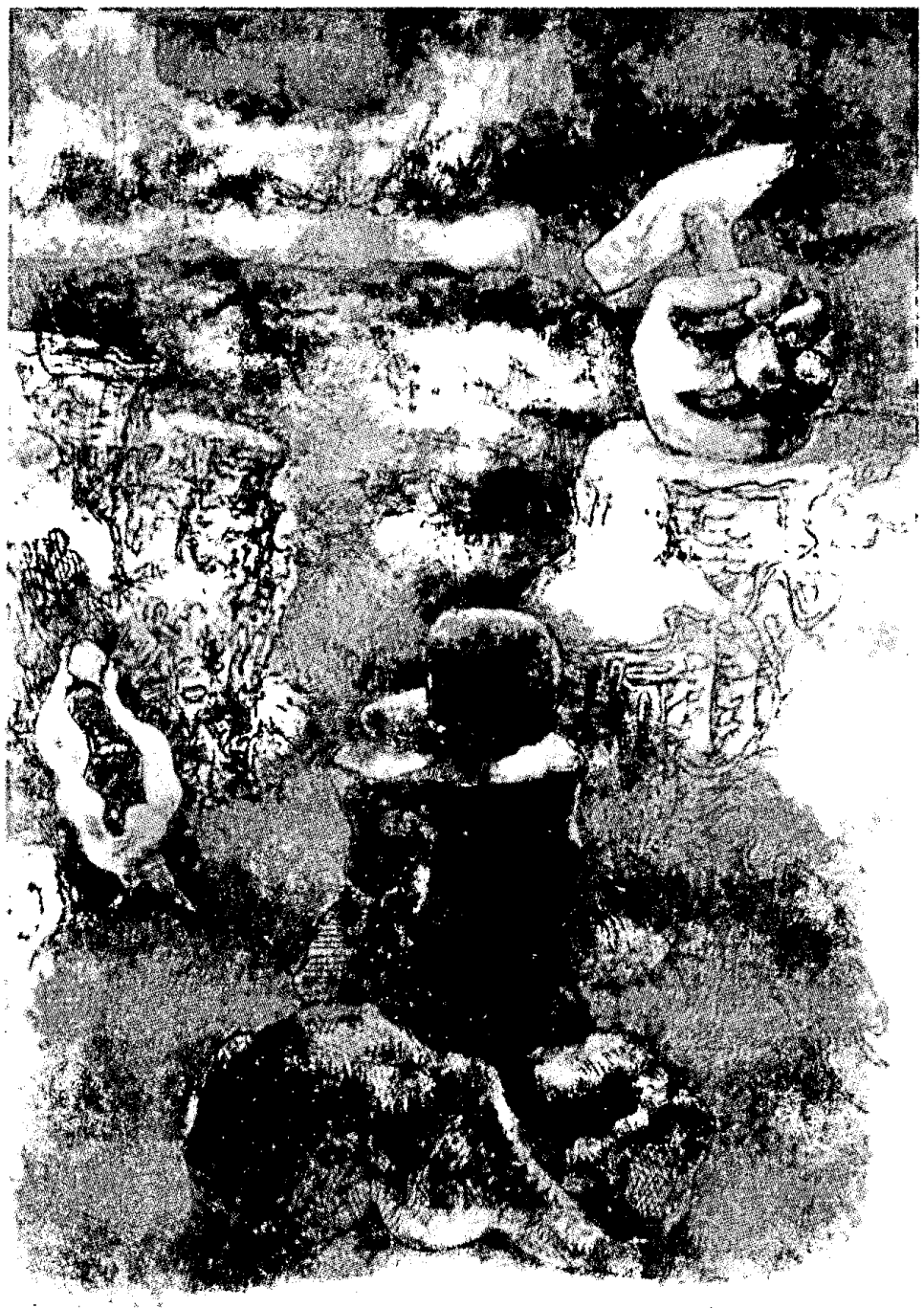


importantes organizaciones que emprendieron una lucha tenaz por la obtención de los principales derechos para las mujeres chilenas, comenzó una fuerte lucha por influir en aquellas esferas de poder que, como el Congreso Nacional, tendrían la última palabra en esta materia.

En este sentido, la primera línea de acción apuntó esencialmente a mejorar la situación de las mujeres dentro del derecho civil. Es así como en 1915 , y posteriormente en 1922, se hicieron presentes dos proyectos tendientes a modificar esta situación; ${ }^{40}$ sin embargo fue hasta 1925 cuando se abolieron las incapacidades civiles más humillantes para las mujeres chilenas. ${ }^{41}$

Por otra parte, la lucha más importante se desarrolló por crear conciencia, en los hombres vinculados al poder, de la necesidad de incorporar a las mujeres al sistema democrático, obteniendo la totalidad de sus derechos políticos. Así, en 1934 las mujeres chilenas obtuvieron el voto municipal, el cual fue el punto de partida de esta larga lucha.

Conforme se fueron fortaleciendo las organizaciones femeniles y se elevaron sus movilizaciones, se fueron sucediendo el número de proyectos en el Congreso Nacional que pretendieron igualar la situación política de mujeres y hombres. De este modo, en 1937 un grupo de políticos denominados "Democracia Unificada" presentó a la Cámara de Diputados un proyecto

${ }^{40}$ El Mercurio de Valparaiso, 18 octubre 1915 y El Diario Ilustrado, febrero-mayo 1922.

41 ElMercurio de Valparaíso, 29 marzo 1925. en tal sentido, que no prosperó. ${ }^{42}$ Otro proyecto fue el presentado al Congreso Nacional por el presidente Pedro Aguirre Cerda en 1941, el cual había sido redactado por las representantes del MEMCH: Elena Caffarena y Flor Heredia. Sin embargo, con la muerte del presidente este proyecto tampoco se concretó en ley de la república. ${ }^{43}$

En 1945, recién fundada la FECHIF, se presentó un nuevo documento que fue definitivo. Dicho proyecto fue patrocinado por nueve senadores de distintas tendencias políticas: Arturo Alessandri Palma, Gustavo Jirón, Marmaduke Grove, E. Amunátegui, Salvador Allende, Rudecindo Ortega, Elías Lafferte, Pedro Opitz y Horacio Walker. ${ }^{44}$ A partir de ese momento, comenzó una ardua discusión en el interior del Congreso Nacional, que se prolongó por casi cuatro años. Dentro de los argumentos más socorridos en favor del logro de este proyecto, estuvieron los compromisos de Chile frente a los tratados y convenios internacionales -Convenios de Chapultepec-; ${ }^{45}$ además, la necesidad de ampliar la base democrática del país incorporando a las mujeres a la vida ciudadana. ${ }^{46} \mathrm{~A}$ diferencia de los proyectos anteriores, éste contaba con el decidido apoyo del movimiento femenil chileno orga-

42 Sesiones Ordinarias del Congreso, Cámara de Diputados, vol. I, 22 junio 1937.

43. Cámara de Diputados, trigésima segunda sesión extraordinaria, 8 enero 1941.

${ }^{44}$ Senado de Chile, sesión novena ordinaria, 20 julio 1945.

45 Senado de Chile, sesión novena ordinaria, 20 julio 1945.

46 Senado de Chile, sesión ordinaria, 29 mayo 1946. 
nizado en la FECHIF, la cual mantuvo importantes relaciones internacionales, lo que permitió un flujo considerable de cablegramas de distintos organismos y personalidades internacionales en favor de los derechos políticos para las mujeres chilenas.

En síntesis, la lucha que las organizaciones femeniles habían emprendido, comenzó a tomar cuerpo; faltaba el impulso final, en las acciones, en los argumentos, en las calles. Faltaba en definitiva, un último empujón para que lo establecido en el oficio que llegó a la Cámara de Diputados el 24 de diciembre de 1946 se convirtiera en ley de la república.

EL. SUFRAGIO: LOGRO DEL MOVIMIENTO FEMINISTA (1947-1952)

...Este nombramiento era la culminación de un largo proceso que vivimos las mujeres chilenas encabezado por la entonces Primera Dama de la nación, doña Rosa Marckman de González Videla. Gracias, fundamentalmente a su tesón y la comprensión de su esposo, elprimer mandatario, en 1949 fue realidad nuestro anhelo: los plenos derechos políticos de la mujer. ${ }^{47}$

La larga lucha emprendida por las mujeres organizadas desde la segunda década del siglo $\mathrm{XX}$, tuvo sus frutos en el periodo que va desde 1947 a 1952 , ya que sus derechos de elegir y ser elegidas por fin fueron conquistados.

\footnotetext{
${ }^{47}$ Adriana Olguín de Baitra-primera ministra de Estado en América-, refiriéndose a su nombramiento. Las cursivas son mías. El Mercurio, 3 marzo 1973, p. 35.
}

La campaña para conquistar tales derechos tomó, entre 1947 y 1949 , un carácter decidido y frontal: el tema se había puesto a la orden del día en la opinión pública y esta razón, además de la politización que vivía el país, impulsó al elemento femenil con opiniones y participación directa en los acontecimientos. Ejemplo de esto fue la vitalidad con que las mujeres enfrentaron las elecciones municipales de 1947 en las que fue elegida, por primera vez, una mujer de izquierda: Julieta Campusano, militante del Partido Comunista; ${ }^{48}$ también lo fue la activa presión que ejercieron las mujeres ante la Cámara de Diputados para conseguir de éstos la pronta promulgación de la ley.

Otro hecho que caracterizó el periodo y que refuerza la visión de creciente participación de las mujeres en política, es el surgimiento de nuevas organizaciones: La Unión de Mujeres de Chile (UMCH) y el Partido Femenino Chileno (fundado un año antes, en 1946). La primera pretendió levantar el movimiento femenil, en franca decadencia una vez conseguido el voto y la segunda, de carácter muy singular debido a las especiales dotes y carisma de su presidenta, María de la Cruz, que deslumbró a la opinión pública con sus encendidos discursos. Debido a ello logró reclutar importantes contingentes de mujeres hasta entonces no organizadas bajo el predicamento de que si bien el papel tradicional de las mujeres era el de esposa y madre, éstas podrían, eventualmente intervenir en política. ${ }^{49}$

${ }^{48}$ El Stglo, 21 abril 1947.

${ }^{49}$ El Mercurio, 2 diciembre 1947. 
Con objeto de agilizar la campaña por el voto surgió el Comité Unido Pro-Voto Femenino, conformado por las más importantes y diversas organizaciones femeniles, las delegadas de todos los partidos políticos, las mujeres independientes y los comités de las estudiantes universitarias. Una frase que ilustra su decisión y manifiesta el deseo por conquistar sus derechos políticos plenos, emanó de una de sus dirigentes: "Hoy por hoy, para nosotras, la mejor galantería debe tener la forma y el sentido de un derecho, el de elegir a nuestros gobernantes". 50

En su conjunto, ninguna de estas tres organizaciones logró perdurar, como lo hicieron el MEMCH y la FECHIF, ni aportar algo significativo a la lucha por la emancipación integral de las mujeres. Su mérito, y en este sentido me refiero, sobre todo, al Partido Femenino Chileno, fue activar la opinión pública y que ésta se hiciera propicia a la concesión del voto para las mujeres. En ese ambiente de evidente sensibilidad frente al tema de los derechos políticos de las mujeres, se firmó en 1949 la ley núm. 9.292, que les confirió el sufragio.

Diversas acciones precedieron a ese importante acto: de hecho el proyecto fue demorado en su aprobación en la Cámara de Diputados, lo que obligó a las organizaciones a presionar a través de actos de presencia masiva ante las autoridades para exigir el cumplimiento del trámite. ${ }^{51}$ Con la consigna "Nosotras somos colaboradoras de los hombres en la vida, queremos

so El Mundo, 10 julio 1947.

${ }^{51}$ El Siglo, 21 mayo 1947. serlo en la política", las mujeres exigieron a los diputados acelerar el trámite de aprobación. Sin embargo, éste demoró dos años desde su aprobación en 1947 por parte del Senado.

Probablemente, la tardanza de los diputados para discutir y aprobar el proyecto se debió al ya tradicional temor de que el mapa electoral se modificara con la intervención femenil, corriendo el riesgo de perder o disminuir sus posiciones. No obstante, tal tardanza, en vez de desanimar a las mujeres, motivó a las organizaciones a realizar movilizaciones con el fin de agudizar su presión. Estas prácticas (concentraciones públicas en calles o teatros) dejaron de manifiesto que las mujeres eran un actor social de importancia y que podían llegar a serlo, también, en política. Por tal motivo, considerar que el Congreso o el presidente de la república concedieron, a modo de regalo, los derechos políticos a las mujeres chilenas, constituiría un error y una injusticia histórica. Por el contrario, a través de sus organizaciones y sus actos de presión (manifestaciones, concentraciones, peticiones públicas, etcétera) y de promoción (charlas y boletines informativos) las mujeres conquistaron su derecho al voto.

$A$ lo anterior debe agregarse que el por entonces presidente Gabriel González Videla (1946-1952) comprendió que, más temprano que tarde, tendría que firmar dicha ley: si no lo hacía, sus bases políticas sufrirían una merma aún mayor de la que sufrió después de la promulgación de la Ley de Defensa de la Democracia, que puso en la ilegalidad al Partido Comunista. Por otra parte, su gobierno quedaría desacre- 
ditado ante los tratados internacionales, contrariaría al Senado y traicionaría su programa político por cuanto en él estaba contenido un capítulo relativo a la concesión de los derechos políticos a las mujeres.

En 1949 se inició una nueva etapa: la del ejercicio de los derechos conquistados; sin embargo, el sectarismo y la falta de compromiso hacia los derechos de las mujeres por parte del gobierno de Gabriel González Videla quedó demostrado: se privó del derecho al voto a Elena Caffarena, abogada y destacada militante del movimiento feminista chileno, a sólo tres días de promulgada la ley, por sus relaciones con miembros del Partido Comunista. Gracias a la solidaridad internacional y a su propia defensa logró reconquistarlo. 52

Las primeras acciones, una vez conquistado el voto, fueron: realizar campañas para conseguir que la masa femenil ejerciera este derecho, empezando por su inscripción en el Registro Electoral. ${ }^{53}$ También, las mujeres ejercieron su derecho a ser elegidas. En 1951 Inés Enríquez Frödden, militante del Partido Radical, fue elegida en la ciudad de Concepción como la primera mujer diputada de nuestro país. En 1952 la ibañista y representante del Partido Femenino Chileno, María de la

\footnotetext{
52 Para más detalles sobre este hecho véase Eltit, Mi derecho, 1993.

${ }_{53}$ Como era de suponer, en un comienzo el número de mujeres inscritas en edad de votar fue reducido en comparación con el de los varones. Así, sólo $19.5 \%$ de las mujeres en edad de votar estaba inscrito para las elecciones presidenciales de 1952. Fuente: Servicio Electoral de Chile.
}

Cruz, se convirtió en la primera senadora. Sin embargo, su actuación no estuvo exenta de numerosas dificultades que derivaron en su inhabilitación. ${ }^{54}$

En ese mismo año, las mujeres chilenas eligieron por primera vez a un presidente de la república. El resultado favoreció a Carlos Ibáñez del Campo (1952-1958).

La etapa del goce de los derechos políticos que tras larga, perseverante e inteligente lucha se logró, inició un nuevo capítulo en la historia del movimiento feminista contemporáneo.

\section{CONCLUSIONES}

Sobre el tema de la obtención y ejercicio de los derechos políticos de las mujeres chilenas, vale la pena recordar que fueron muchos los factores que influyeron en ese proceso. Algunos, como la industrialización y la expansión urbana, ${ }^{55}$ vinculados con el cambio estructural de la economía entre 1880 y 1930,56 posibilitaron a las mujeres el contacto con modos de vida alternativos y su incorporación al trabajo asalariado. Otros, como la promulgación del decreto Amunátegui en 1877 , les permitió obtener educación superior. Por su parte, la participación en las luchas de los sectores obreros por mejores condiciones de vida?

${ }^{54}$ Gaviola et al., "Queremos", 1986.

55 "By the end of the 1920 s almost 50 percent of the population was urban in character". Lavrin, Journal, 1989, p. 89.

56 Explotación de grandes recursos salitreros obtenidos después de la guerra del Pacífico (1879) y posterior sustitución de importacio. nes.

57 Como ejemplo podemos señalar la partici- 
y la influencia de pensadores que propugnaban por la igualdad (como John Stuart Mill) fueron propiciando lentamente el ascenso del movimiento sufragista.

En la misma esfera, la influencia de los medios de comunicación referida a los avances del feminismo. a nivel mundial, la visita de conferencistas extranjeros que difundieron el libre pensamiento y la ideología feminista en Chile, así como los cambios originados por la primera guerra mundial (1914-1918) y la crisis capitalista de 1929-30, favorecieron el despertar de la conciencia feminista chilena en el primer tercio del siglo $\mathrm{xx} .{ }^{58}$ Dicha conciencia se materializó con el surgimiento de innumerables organizaciones femeniles de diverso carácter, ${ }^{59}$ cada una de las cuales, en distintos momentos, aportó al proceso de emancipación de las mujeres chilenas.

La suma de estos factores coadyuvó al nacimiento de la conciencia feminista y determinó que en 1949 las mujeres chilenas obtuvieran su más preciada conquista: el derecho al sufragio pleno, que habían comenzado a demandar desde el siglo XIX.

Finalmente, quisiera resumir las principales características del movimiento feminista en Chile: una primera etapa sufragista desde los primeros años del siglo $\mathrm{XX}$ hasta la consecución del derecho al voto en 1949 . Se trató de un movimiento liderado por mu-

pación de las mujeres en la zona del salitre apoyando las huelgas de los trabajadores.

${ }^{58}$ Gaviola et al., "Queremos", 1986.

59 La masificación del movimiento femenil chileno a partir de 1935 sevio favorecida por el desarrollo de las luchas sociales en el país. jeres educadas, profesionales de clase media y sectores de la clase alta ilustra$\mathrm{da}$, quienes reivindicaron la igualdad de las mujeres dentro de la sociedad y cuyo principal objetivo fue la obtención de los derechos políticos plenos.

La promulgación de la ley núm. 9.292 permitió a las mujeres votar en elecciones parlamentarias y presidenciales gracias a una larga y progresiva lucha de las organizaciones femeniles y a la necesidad del por entonces presidente de Chile, Gabriel González Videla(1946-1952), de contar con nuevos adeptos para su malogrado gobierno.

La tardanza con que se produjo la obtención de los derechos políticos se explica, en gran medida, por la incertidumbre de los partidos de la época respecto del futuro comportamiento electoral de las mujeres. ${ }^{6}$

Una vez que se obtuvo el derecho al voto y las mujeres participaron en las primeras elecciones presidenciales (1952), siguió una segunda etapa caracterizada por la desintegración del movimiento. Este proceso se debió a la imposibilidad de conseguir una unidad perdurable entre mujeres de diversas clases sociales, con distintas necesidades $y$ aspiraciones, y a la plena vigencia de una ideología sexista que, si bien no pudo impedir a las mujeres

${ }^{60}$ El comportamiento político conservador mostrado por las mujeres en las primeras elecciones municipales, pariamentarias y presidenciales en que les tocó participar se debería, más que a un rasgo inherente a la "psicologia feme. nina", a la habilidad de los partidos de derecha para atraerlas y utilizarlas políticamente, y a la indiferencia de la izquierda, que dejó al movimiento femenil entregado a su propia suerte. Gaviola et al., "Queremos", 1986. 


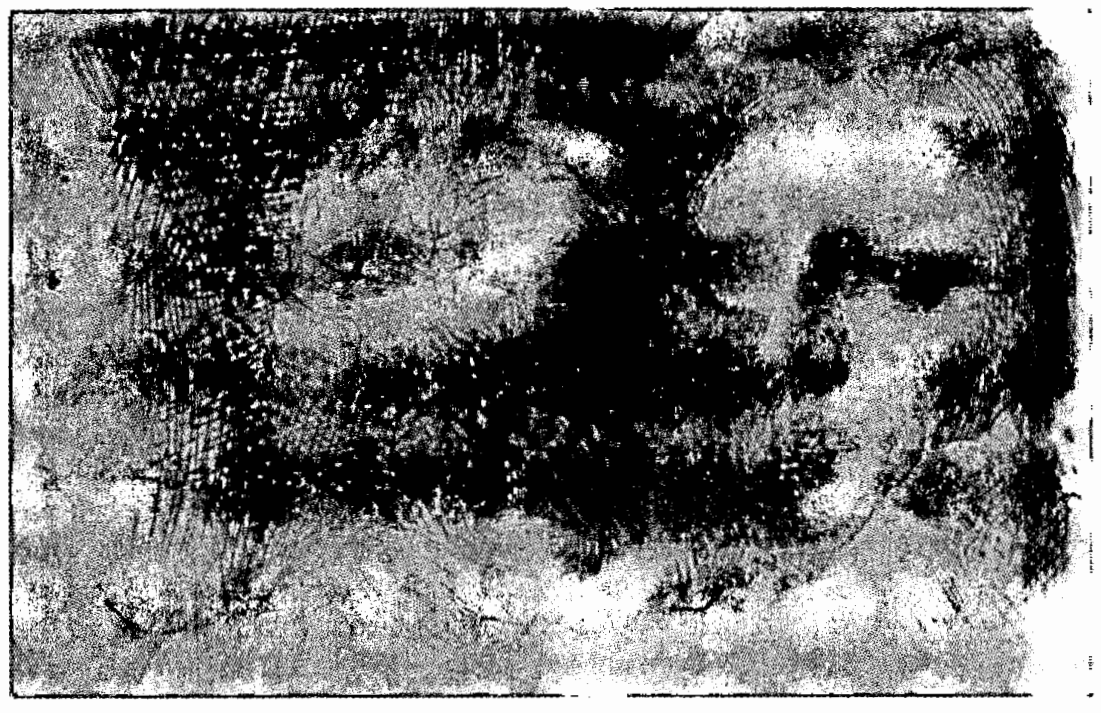

la obtención de ciertos derechos, no les permitió ejercerlos en forma igualitaria con los hombres. Por último, la conciencia de las propias organizaciones femeniles subordinó al problema político otros aspectos de la opresión de las mujeres, tan importantes como el anterior.

A la desintegración del movimiento siguió la organización de las mujeres en instituciones, incorporándose éstas a partidos políticos, sindicatos y movimientos populares, desde donde participaron en calidad de miembros de una clase social y en el cual fueron escasas las demandas de género. Si bien en este lapso no hubo un movimiento feminista organizado de carácter masivo, sí se produjo la inserción de la mujeres en la política, tanto en los partidos como en el aparato estatal, a través de cargos de representación. Las mujeres que obtuvieron cargos públicos y otras militantes de partidos políticos tendieron a una corriente del feminismo llamada liberal, que planteó cambios en la legislación y en el marco jurídico, reivindicando la igualdad social, política, económica y educacional. Una tercera etapa, a partir de mediados de la década de los setenta, estuvo caracterizada por el desarrollo en Chile del llamado feminismo contemporáneo, que incluyó las corrientes radical y socialista ${ }^{61}$ y que reivindicó la igualdad aunque tam-

61 Para más detalles sobre las distintas etapas del movimiento feminista y corrientes del feminismo, véase Ungo, "Subordinación", 1992. 
bién cuestionó las relaciones entre los géneros dentro de la familia identificándose con la consigna de los años setenta "lo personal es político", y creando instituciones autónomas de mujeres que estructuraron un movimiento sociopolítico que fue desarrollando a través de la praxis una rica discusión teórica. También correspondió a esta tercera etapa la movilización y la participación masiva de las mujeres en la coyuntura política (dictadura militar, 1973-1990), así como la reivindicación de demandas de género e integración del movimiento feminista chileno a las iniciativas internacionales. ${ }^{62}$

Cronología de Los Hechos

MÁS IMPORTANTES DE LA HISTORIA

DE LAS MUJERES CHILENAS

(1877-1953)

1877. Publicación del Decreto Amunátegui, que permite el ingreso de las mujeres a la Universidad. Luchan por esta iniciativa las maestras normalistas Antonia Tarragó e Isabel Le Brun.

1884. Un grupo de mujeres de la ciudad de San Felipe intenta hacer efectivo el derecho al voto consagrado en la Constitución (1833), tratando de inscribirse en los registros electorales.

1887. Se reciben de médico Ernestina Pérez y Eloísa Díaz, siendo ambas las primeras de toda Iberoamérica.

62 Participación en el decenio de la mujer (1975-1985) y en el Primer Encuentro Feminista Latinoamericano y del Caribe en 1981. Ungo, "Subordinación", 1992, pp. 124 y 127.
1902. Primera profesora de Historia y Geografia (Zenobia Arratia).

1913. Fundación del Centro Femenino Anticlerical Belén de Sárraga, bajo la inspiración de Luis Emilio Recabarren y 'Teresa Flores.

1915. Fundación del Círculo de Lectura y del Club Social de Señoras.

1925. Concesión de derechos civiles a las mujeres (la madre obtuvo la patria potestad al morir el padre, la mujer podía ser testigo en un juicio $y$ tutora de menores, pero con autorización del marido si era casada).

1931. Primera movilización femenil de índole política. Se funda la Asociación de Mujeres Universitarias.

1934. Se promulga la ley núm. 5.357, que le otorga a las mujeres el derecho a elegir y ser elegidas en elecciones municipales.

1935. Fundación del Movimiento ProEmancipación de la Mujer Chilena (MEMCH). Arribo de las mujeres al municipio.

1936. Primera jueza.

1941. Las feministas Elena Caffarena y Flor Heredia redactan un proyecto de ley sobre sufragio femenil, presentándolo al por entonces presidente, Pedro Aguirre Cerda.

1944. Primer Congreso Nacional de Organizaciones Femeninas. Fundación de la Federación Chilena de Instituciones Femeninas (FECHIF).

1945. Se otorga el Premio Nobel de Literatura a la poetisa Gabriela Mistral (el primer Nobel de Latinoamérica).

1946. Fundación del Partido Femenino Chileno. Primera ministra de Estado (Adriana Olguín), en el gobierno de Gabriel González Videla 
Secuencia (1996), 36, septiembre-diciembre, 245-248

ISSN: 0186-0348, ISSN electrónico: 2395-8464

DOI: http://dx.doi.org/10.18234/secuencia.v0i36.553

(1946-1952). Primera embajadora de la República (Amanda Labarca). 1947. Fundación de la "Asociación de Dueñas de Casa".

1949. Obtención del voto político para las mujeres por la Ley núm. 9.292 (elecciones parlamentarias y presidenciales).

1950. Primera diputada de la república (Inés Enríquez Frödden). Fundación de la Unión de Mujeres de Chile.

1952. Primera elección presidencial con participación femenil.

1953. Fundación de la Central Única de Trabajadores (CUT) a la cual se integraron las mujeres como trabajadoras sindicalizadas o como diri gentas. Primera senadora de la república (María de la Cruz Toledo).

\section{BIBLIOGRAFÍA}

-Bonder, Gloria, "El estudio de la política desde la perspectiva de las mujeres", $R e$ vista Internacional de Ciencias Sociales: La mujer y las esferas de poder, UNESCO, núm. 4, vol. Xxxv, 1983.

- Caffarena, Elena, Un capítulo en la historia del feminismo, МЕМСH; Santiago de Chile, 1952.

-Cano, Gabriela y Verena Radkau, "Lo privado y lo público o la mutación de los espacios. (Historias de mujeres 19201940)", en Vania Salles y Elsie McPhail (comps.), La investigación sobre la mujer: informes en sus primeras versiones, PIEM/El Colegio de México, México, 1988 (Documentos de Investigación, 1).

-Chaney, Elsa, Supermadre, Fondo de Cultura Económica, México, 1983.

-Covarrubias, Paz y Rolando Franco, Chile, mujer y sociedad, Alfabeta Impresores, Santiago de Chile, 1978.
-De Barbieri, Teresita, Movimientos feministas, Coordinación de HumanidadesUNAM, México, 1986 (Grandes Tendencias Políticas Contemporäneas/37). , y Orlandina De Oliveira, "Nuevos sujetos sociales: la presencia política de las mujeres en América Latina", Nueva Antropología, núm. 30, vol. viII, 1986, México.

-De Lauretis, Teresa, "Estudios feministas/Estudios críticos: problemas, conceptos y contextos", en Carmen Ramos Escandón (comp.), El género en perspectiva: de la dominación universal a la representación múltiple, UAM-lztapalapa, México, 1991.

-Del Valle Stark, María, "Mujer. Legislación 1810-1984", Revista de Legislación y Documentación en Derecho y Ciencias Sociales, año vI, núm 4, octubre-diciembre, 1984, Santiago de Chile.

-Dietz, Mary G., "El contexto es lo que cuenta: feminismo y teorías de la ciudadanía", Debate feminista, año 1, vol. 1, 1990.

-Duby, Georges y Michelle Perrot, Historia de las mujeres en occidente, Taurus, Madrid, 1991, 5 vols.

-Eltit, Diamela, Mi derecho a voz, mi derecho a voto, Casa de Chile, México, 1993.

-Foucault, Michel, Microfísica del poder, Ediciones de la Piqueta, Madrid, 1979.

-Gallego Méndez, María Teresa, "Notas sobre el poder, la socialización política y la mujer (la sección femenina de la Falange)", en Nuevas perspectivas sobre la mujer, vol. II, tercera parte, $\mathbf{s} /$ f.

-Gaviola, Edda, Ximena Jiles, Lorella Lopresti y Claudia Rojas, "La mujer chilena como fenómeno político de nuestro tiempo, 1913-1952", IV Encuentro de Historiadores Jóvenes, Santiago de Chile, Facultad Latino Americana de Ciencias Sociales (FLACSO), 10 a 12 de abril de 1986, mimeo. "Queremos votar en las próximas elecciones", en Historia del movimiento femenino chileno (1913-1952), 
Secuencia (1996), 36, septiembre-diciembre, 245-248

ISSN: 0186-0348, ISSN electrónico: 2395-8464

DOI: http://dx.doi.org/10.18234/secuencia.v0i36.553

CEM/Fempress/ISIS-Internacional/Librería Lila/La Morada, Santiago de Chile, 1986.

, Nuestra historia de mujeres (cuadernillo de educación popular), Santiago de Chile, Ediciones La Morada, 1988.

-Gran Sopena, Diccionario Ilustrado de la lengua española, Ramón Sopena, Barcelona, 1980,3 vols.

-Hutchison, Elizabeth, "El feminismo en el movimiento obrero chileno: la emancipación de la mujer en la prensa obrera feminista, 1905-1908", en Proposiciones, núm. 21, Ediciones Sur, 1993, Santiago de Chile.

-Kirkwood, Julieta, "La política del feminismo en Chile", en Revista Internacional de Ciencias Sociales: La mujer y las esferas de poder, UNESCO, núm. 4, vol. XXXV, 1983. feministas y los partidos, FLACSO, Santiago de Chile, 1984.

"Chile: La mujer y la formulación política", en Revista Signos, AGECH, 1984, Santiago de Chile.

-Klimpel, Felícitas, La mujer chilena: el aporte femenino al progreso de Chile 1910-1960, Andrés Bello, Santiago de Chile, 1962.

-Labarca, Amanda, Feminismo contem. poráneo, Zig-Zag, Santiago de Chile, 1947.

-Lamas, Marta, "La antropología feminista y la categoria género", en Nueva Antropología, núm. 30, vol. vII, 1986, México.

Lavrín, Asunción (comp.), Las muje. res latinoamericanas. Perspectivas históricas, Fondo de Cultura Económica, México, 1985.

"Women, labor and the left: Argentina and Chile, 1890-1925", en Jour. nal of Women's History, núm. 2, 1989.

-Maino V., T. Pereira, I. Zegers y L. Santa Cruz, Tres ensayos sobre la mujer chile. na, Universitaria, Santiago de Chile, 1978.

-Mansbridge, Joan, "Feminismo y democracia", en Mujeres en acción, Isis Internacional, núm. 1, 1992, Santiago de Chile.
-McGee Deutsch, Sandra, "Gender and sociopolitical change in twentieth-century Latin America", en Hispanic American Historical Review, Duke University Press, 71, núm. 2, Durham, 1991.

-Ramírez Saavedra, Beatriz, "Feminismo y democracia", Debate feminista, año 3, vol. 5, México, 1992 ,

-Rubin, Gayle, "El tráfico de mujeres: notas sobre la 'economía política' del sexo", Nueva Antropología, núm. 30, vol. VIII, 1986.

- Organización de Estados Americanos (OEA), Comisión Interamericana de Mujeres, 1965.

- Olea, Raquel, "El feminismo ¿moderno o postmoderno?", Mujeres en acción, Isis Internacional, núm. 1, 1991, Santiago de Chile.

Pateman, Carole, "Feminismo y democracia", Debate feminista, año 1, vol. 1, 1990, México.

-Poblete, Olga, "El MEMCH. Un capítulo del militantismo femenino chileno", $R e$ vista Araucaria, 1983.

-Radkau, Verena, "Hacia una historiografía de la mujer", Nueva Antropología, núm. 30, vol. VIII, 1986.

-Ramos Escandón, Carmen, "Las mujeres latinoamericanas: generación de datos y metodología para investigaciones futuras", Secuencia, Instituto Mora, vol. 6, septiembre-diciembre, 1986, México.

-Rosetti, Josefina, "La educación de las mujeres en Chile contemporáneo", en Mundo de mujer, continuidad y cambio, Centro de Estudios de la Mujer (CEM), Santiago de Chile, 1988.

-Salinas, Cecilia, La mujer proletaria: una historia para contar, Literatura Americana Reunida(LAR), Santiago de Chile, 1987.

-Stoner, Lynn, "Directions in Latin American Women's History, 1977-1985", Latin American Research Review, núm. 2, vol. XXII, 1987.

-Thompson, Edward, Miseria de la teoría, Crítica, Grijalbo, Barcelona, 1981. 
Secuencia (1996), 36, septiembre-diciembre, 245-248

ISSN: 0186-0348, ISSN electrónico: 2395-8464

DOI: http://dx.doi.org/10.18234/secuencia.v0i36.553

-Vargas, Virginia, "El poder en cues: tión", Revista Feminista Viva, núm. 7, año 3, septiembre-octubre, 1986.

Tesis referidas a las mujeres

-Gaviola, Edda, Ximena Jiles, Lorella Lopresti y Claudia Rojas, "Evolución de los derechos políticos de la mujer en Chile (1913-1952)", tesis de licenciatura, Universidad de Santiago de Chile, Santiago de Chile, agosto de 1985.

-Ungo, Urania, "Subordinación genérica y alienación política: el discurso de las organizaciones de mujeres de la región centroamericana", tesis de maestría en Estudios Latinoamericanos, Facultad de Filosofia y Letras-UNAM, 1992.

\section{HEMEROGRAFIA}

-Diarios (1910-1952).

- El Mercurio de Santiago.

-El Mercurio de Valparaíso.

-ElDespertarde los Trabajadores (Iquique).
-La Opinión.
- El Siglo.
- El Diario Ilustrado.
-El Mundo. 